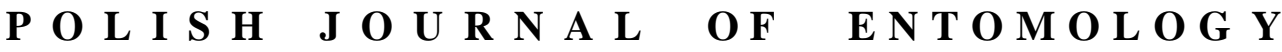

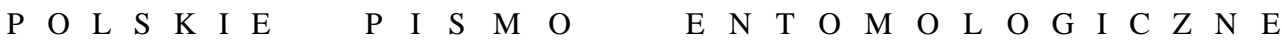

VOL. 84: $325-338$

Lublin

30 December 2015

DOI: $10.1515 /$ pjen-2015-0028

\section{Aphids (Hemiptera: Aphidomorpha) of the Botanic Garden of the Jagiellonian University, Kraków*}

\author{
MARZENA STAROWICZ, MARIUSZ KANTURSKI ${ }^{1}$, ŁUKASZ JUNKIERT ${ }^{2}$, \\ KARINA WIECZOREK ${ }^{3}$
}

Department of Zoology, Faculty of Biology and Environmental Protection, University of Silesia, Bankowa 9, 40-007 Katowice, Poland,

e-mails: mkanturski@us.edu.pl ${ }^{1}$, lukasz.junkiert@wp.pl ${ }^{2}$, karina.wieczorek@us.edu.pl ${ }^{3}$

\begin{abstract}
The paper presents results of faunistic investigations of aphids (Aphidomorpha) in the Botanic Garden of the Jagiellonian University, Kraków, in the Kraków - Wielun Upland. During two seasons of research $(2011,2012)$ two aphid species from the family Adelgidae and 50 species from the family Aphididae, associated with 66 host plants were recorded. The following species - Eriosoma anncharlotteae DANIELSSON, 1979, Capitophorus elaeagni (DEL GUERCIO, 1894), Rhopalosiphoninus (Neorhopalosiphoninus) staphyleae staphyleae (KocH, 1854), Eulachnus brevipilosus BÖRNER, 1940 and E. cembrae BÖRNER, 1950 - are new for the Kraków Wielun Upland. Seven of the species recorded are regarded as alien to Poland.
\end{abstract}

KEY WORDS: aphids, botanical garden, faunistic study, Kraków - Wieluń Upland.

\section{INTRODUCTION}

Botanic gardens are classified as green areas and, along with parks, are the oldest form of urban greenery. In addition to parks and gardens, urban green areas include allotments, cemeteries, sports grounds, roadside vegetation and hedges - artificial creations designed for recreational, aesthetic and educational purposes (WALKOWICZ 2001).

\footnotetext{
* The paper is dedicated to Prof. Wacław WOJCIECHOWSKI in recognition of his great contribution to the taxonomy and faunistics of Hemiptera.
} 
Established in 1783, the Jagiellonian University's Botanic Garden is situated in the Wesoła district of Kraków. The Garden, of the French Baroque type, was originally situated in the valley of the River Wisła (Vistula) and occupied an area of about 2.4 ha (LANKOsZMRÓz 2006). At the present time, the Kraków Botanic Garden ( 9.6 ha in area) is the oldest garden of its kind in Poland with a very large variety of plant species, usually from very distant lands. One can therefore assume that its insect fauna is rich, too.

To date, aphid research in the Jagiellonian University's Botanic Garden has only been done on aphids associated with potted and greenhouse plants (ACHREMOWICZ 1978, ACHREMOWICZ et al. 1986); some aspects of the population dynamics of four aphid species feeding on shrubs have also been reported (WOJCIECHOWICZ-ŻYTKO \& JANKOWSKA 2011). An aphid species new to the Polish fauna - Periphyllus californiensis californiensis (SHINJI, 1917) - considered to be an alien and invasive species, was also found there (JUNKIERT et al. 2011).

The study area consists of diverse plant communities from around the world. The aim of this research was to ascertain the number of aphid species (including alien ones) infesting plants in the Botanical Garden and to assess whether the species richness of aphids increase in parallel with the increase in the number of plant species in a relatively small area.

\section{Acknowledgements}

The authors are very grateful to Prof. dr hab. Bogdan ZEMANEK, the former Director of the Botanic Garden in Kraków, for permission to conduct research in the Garden. We are very grateful to the anonymous Reviewers and the Editor for all their valuable suggestions for improving the manuscript.

\section{MATERIAL AND METHODS}

This faunistic study of aphids in the Botanic Garden of the Jagiellonian University, Kraków took place from 15 April to 15 November in 2011 and 2012. The aphids were collected directly from their host plants; plants in greenhouses were not included in the research. After preservation in 70\% ethanol, the material was prepared after KANTURSKI \& WIECZOREK (2012) and identified to species level. Names and classification follow NIETO NAFRÍA \& FAVRET (2011). The aphid material is deposited in the collection of the Department of Zoology, University of Silesia, Katowice, Poland (UŚ).

To discover the number of alien species, we used the lists of Alien Aphididae in Europe and in Poland, respectively drawn up by COEUR D'ACIER et al. (2010) and WIECZOREK (2011). Trophic relations and chorology are characterized according to WIECZOREK (2010). 
Abbreviations: * - species new to the Kraków - Wieluń Upland; ! - species alien to Poland.

\section{RESULTS}

During the two seasons of faunistic research in the Jagiellonian University's Botanical Garden 52 aphid species (Table 1) were recorded on 66 host plants (Table 2). The aphids belong to two families within Aphidomorpha: Adelgidae and Aphididae. Adelgidae is represented by two species, and Aphididae by 50 species belonging to eight subfamilies: Eriosomatinae, Anoeciinae, Drepanosiphinae, Calaphidinae, Phyllaphidinae, Chaitophorinae, Aphidinae and Lachninae (Fig. 1). Aphidinae, the most numerous subfamily, was represented by 15 genera and 26 species. The richest in species were the genera Aphis LINNAEUS, 1758, Rhopalosiphum KoCH, 1854 (Aphidini) and Brachycaudus VAN DER GOOT, 1913 (Macrosiphini) (Fig. 2). The subfamily Calaphidinae (11 species) was most frequently represented by species belonging to the tribe Panaphidini; the species most often recorded were from the genus Myzocallis PASSERINI, 1860 (Fig. 3). The fewest species were from the subfamilies Anoeciinae, Drepanosiphinae and Phyllaphidinae (Fig. 1). Chorological analysis showed that most species have a worldwide or Holarctic distribution (Fig. 4). Trophic analysis showed that the aphids collected in this Botanic Garden are mainly $1^{\text {st }}$ degree monophagous or oligophagous (Fig. 5). Five species were recorded for the first time in the Kraków - Wielun Upland, and seven are regarded as alien to the Polish aphid fauna (Table 1).

\section{DISCUSSION}

Previous studies in the Botanic Garden of the Jagiellonian University, conducted in greenhouses and focused mainly on the aphids of potted plants, yielded 12 species of aphids (ACHREMOWICZ 1978, ACHREMOWICZ et al. 1986). The present study in 2011 and 2012 did not include greenhouses; only Rhopalosiphum nymphaeae among the species listed by ACHREMOWICZ was confirmed. Research into the population dynamics of four species of aphids - Aphis (Aphis) fabae, Cryptomyzus ribis (LINNAEUs, 1758), Rhopalosiphum padi and Liosomaphis berberidis - was also conducted in this Botanic Garden (WOJCIECHOWICZ-ŻYTKO \& JANKOWSKA 2011). Of these species, only Cryptomyzus ribis was not observed during the present study. Periphyllus californiensis californiensis was also confirmed, as was the occurrence of oviparous females and winged males of this species. Sexual morphs were not identified in previous studies (JUNKIERT et al. 2011). 


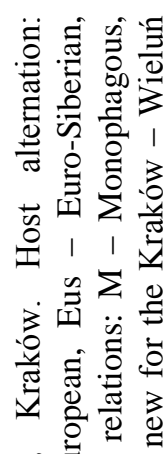

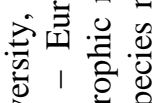
: $\exists$ के 当至

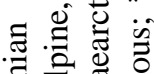
윤 $\frac{0}{50}, \frac{2}{2}$

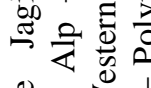
$\stackrel{0}{ \pm} \sum^{0}$

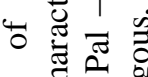
륭 उ 范 . 苍

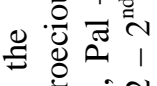

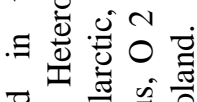
닝

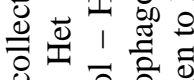

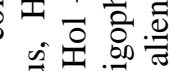
흘

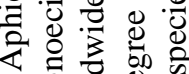
.

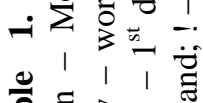

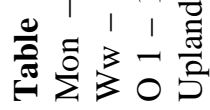

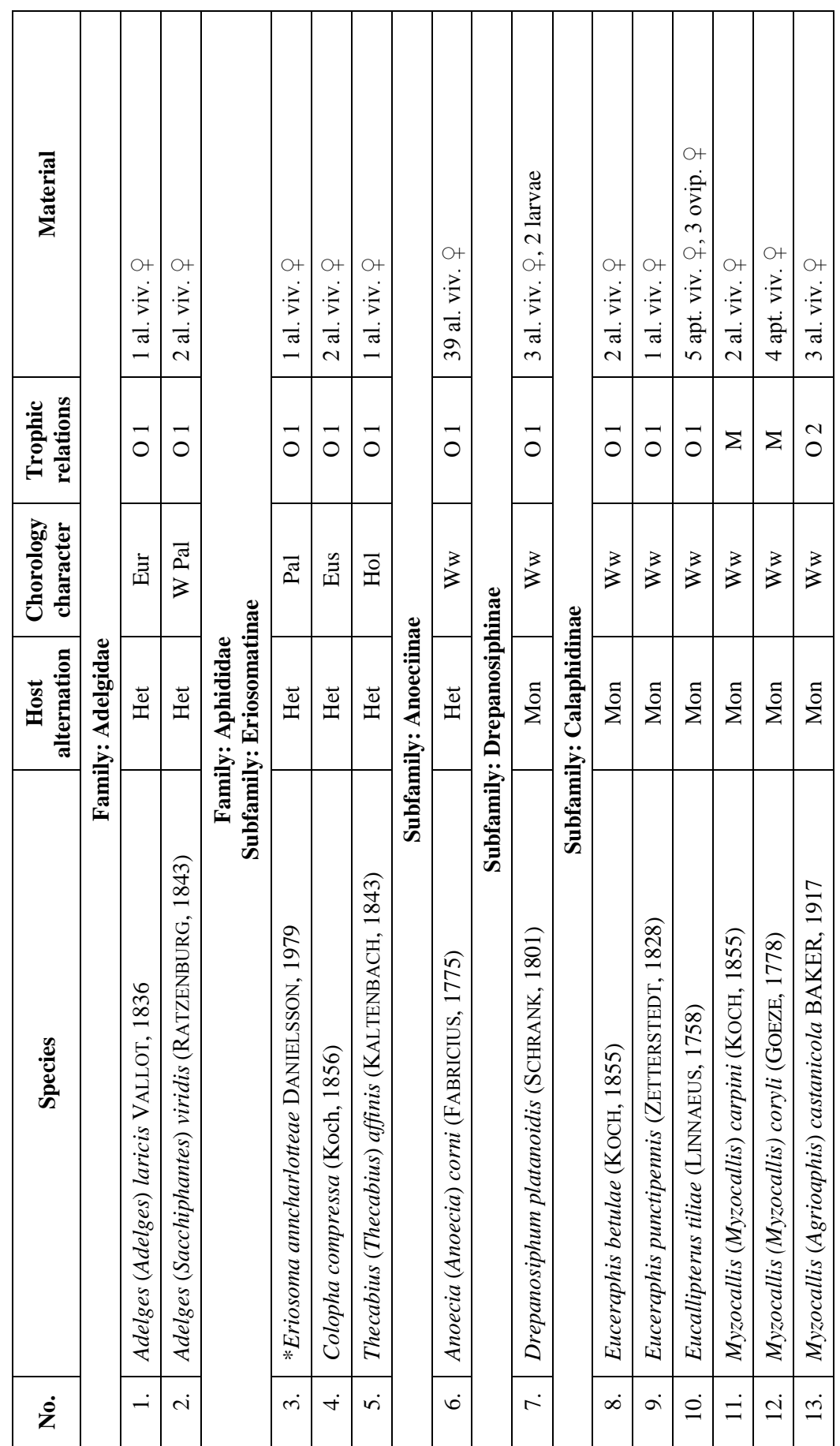




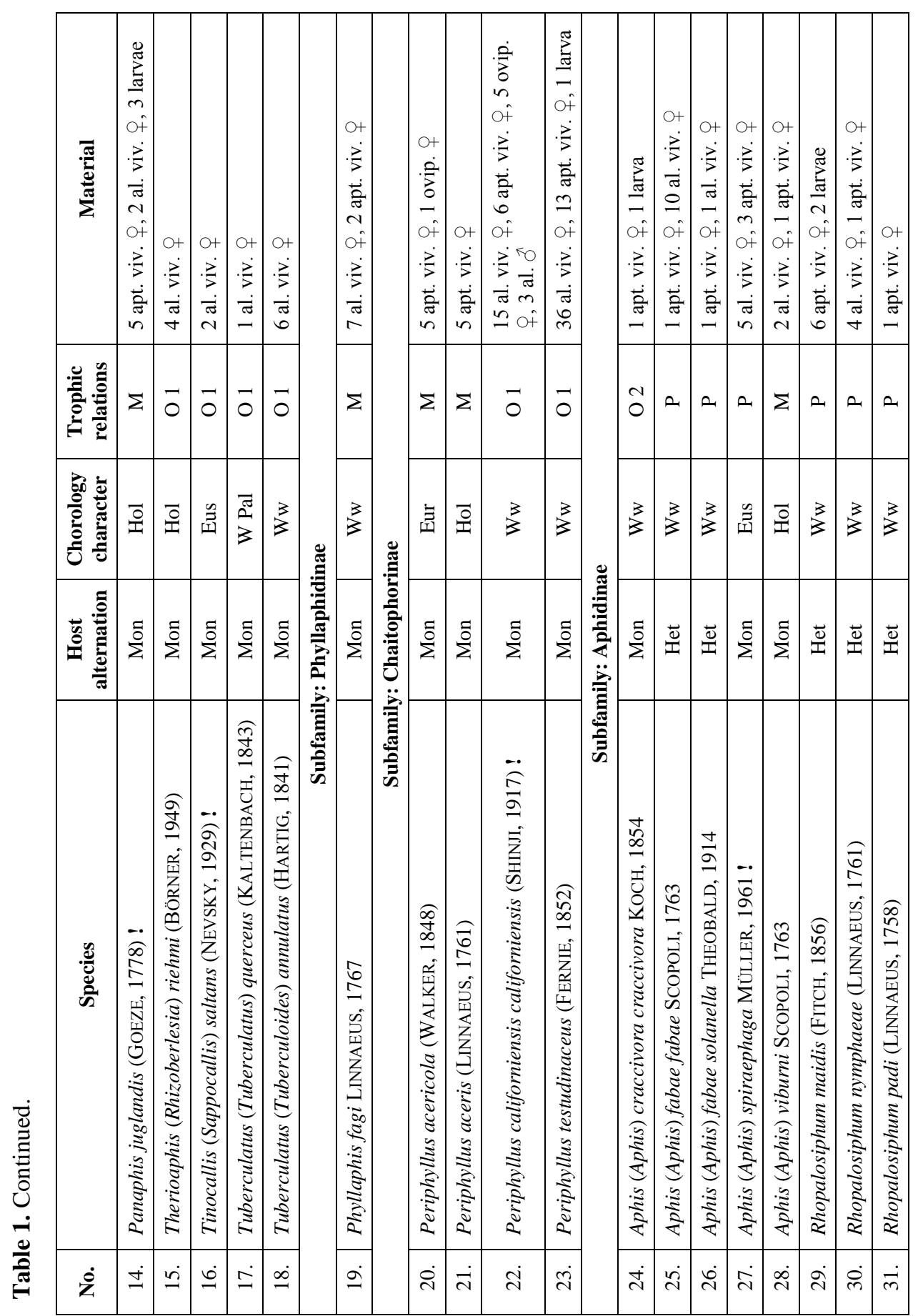




\begin{tabular}{|c|c|c|c|c|c|c|c|c|c|c|c|c|c|c|c|c|c|c|}
\hline 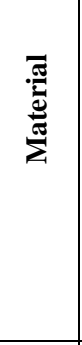 & 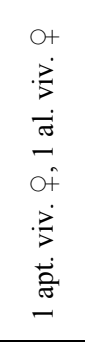 & 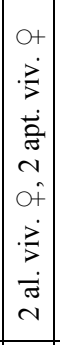 & 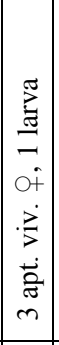 & 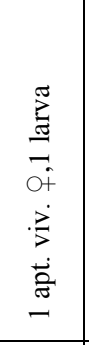 & 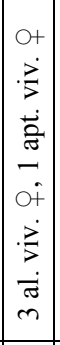 & 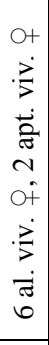 & $\begin{array}{l}\text { O+ } \\
\dot{\vec{j}} \\
\overrightarrow{\vec{a}} \\
\stackrel{\vec{\sigma}}{\mathrm{N}}\end{array}$ & 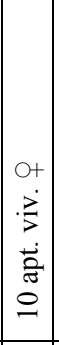 & 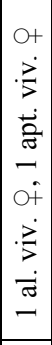 & 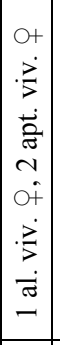 & 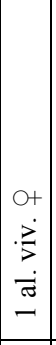 & $\begin{array}{l}\text { O+ } \\
\dot{\vec{z}} \\
\dot{\vec{a}} \\
\dot{\vec{\sigma}} \\
\vec{n}\end{array}$ & 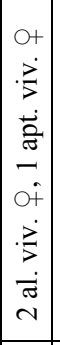 & 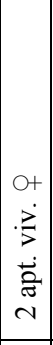 & 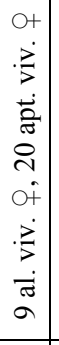 & $\begin{array}{c}\text { ot } \\
\dot{\vec{z}} \\
\dot{\vec{z}} \\
\dot{\vec{a}} \\
\vec{\sigma} \\
\dot{m}\end{array}$ & \begin{tabular}{|l|} 
\\
\\
$0+$ \\
$\dot{\vec{j}}$ \\
$\dot{\vec{j}}$ \\
$\dot{\vec{\sigma}}$ \\
$\mathrm{m}$
\end{tabular} & $\begin{array}{l}\text { O+ } \\
\dot{\vec{j}} \\
\dot{\vec{\sigma}} \\
\overrightarrow{-}\end{array}$ \\
\hline 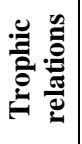 & $\tilde{o}$ & $\Sigma$ & $\begin{array}{l}2 \\
\vdots \\
0\end{array} \mid$ & $\overline{0}$ & $\begin{array}{l}0 \\
0 \\
-1 \\
0\end{array}$ & $\tilde{O}$ & $\overline{0}$ & $\Sigma$ & $\overrightarrow{0}$ & $\overrightarrow{0}$ & $\Sigma$ & $\overrightarrow{0}$ & $\tilde{O}$ & $\overrightarrow{0}$ & $\overline{0}$ & 2 & $\overline{0}$ & 2 \\
\hline 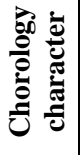 & $3^{3}$ & 壱 & $\stackrel{0}{1}$ & $3^{3}$ & $3^{3}$ & $\begin{array}{l}\overline{0} \\
3 \\
3\end{array}$ & $3^{3}$ & $3^{3}$ & $3^{3}$ & $3^{3}$ & 言 & 定 & $\bar{\sigma}$ & $3^{3}$ & $3^{3}$ & $3^{3}$ & $\overrightarrow{0}$ & 홈 \\
\hline 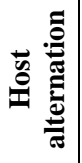 & $\stackrel{\sigma}{\Sigma}^{2}$ & $\sum^{\tilde{D}}$ & $\overrightarrow{\vec{\Xi}}$ & $\stackrel{0}{\Sigma}^{\tilde{n}}$ & $\stackrel{\vec{\Xi}}{\mathbb{I}}$ & $\stackrel{\vec{\Xi}}{\underline{I}}$ & $\stackrel{\delta}{\Sigma}$ & $\stackrel{\tilde{\Xi}}{\Sigma}$ & $\overrightarrow{\widetilde{\Xi}}$ & 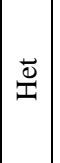 & 芩 & 金 & $\stackrel{\tilde{\Xi}}{\Sigma}$ & $\sum^{0}$ & $\stackrel{\vec{\Xi}}{\Psi}$ & 离 & 矛 & $\stackrel{\vec{\Xi}}{\mathbb{Z}}$ \\
\hline 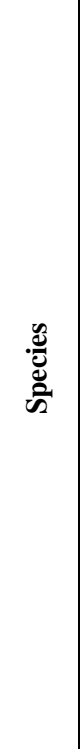 & 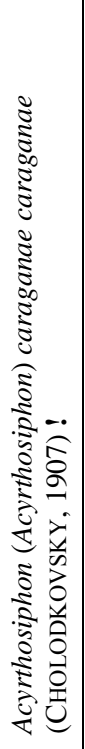 & 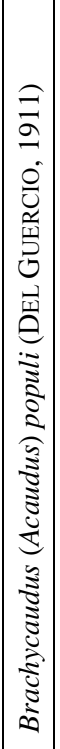 & 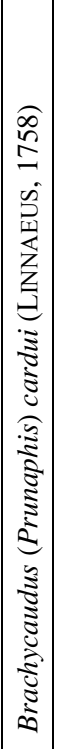 & 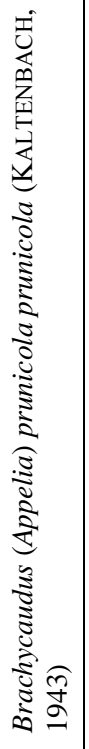 & 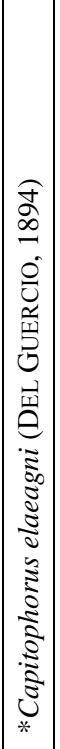 & 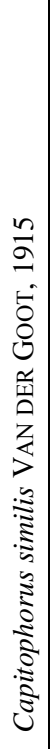 & 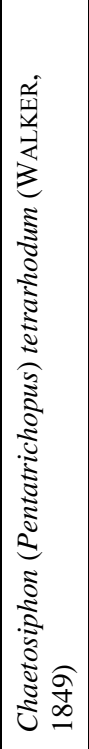 & 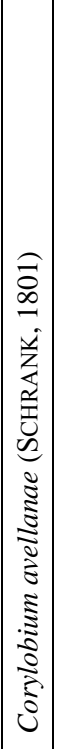 & 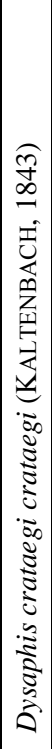 & 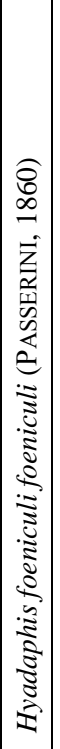 & 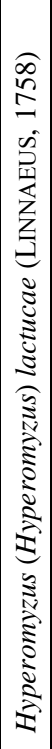 & 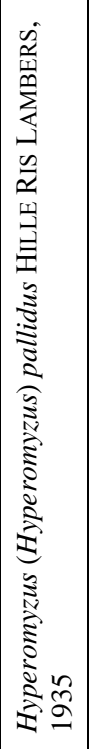 & 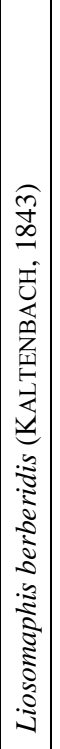 & 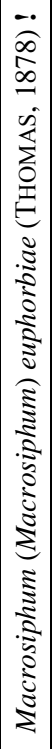 & 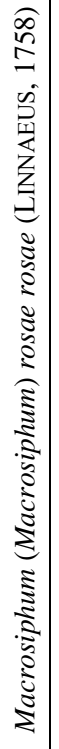 & 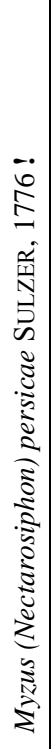 & 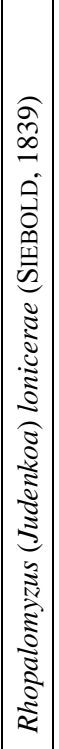 & 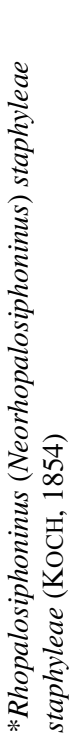 \\
\hline$\dot{\mathbf{z}}$ & लં & लें & $\dot{m}$ & $\ddot{n}$ & $\dot{ల}$ & $\dot{m}$ & $\stackrel{\infty}{m}$ & $\dot{m}$ & $\dot{q}$ & $\dot{\forall}$ & $\stackrel{\sim}{\mathrm{f}}$ & $\dot{q}$ & $\dot{f}$ & 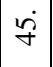 & $\dot{q}$ & $\dot{q}$ & $\stackrel{\infty}{\stackrel{\infty}{o}}$ & $\dot{q}$ \\
\hline
\end{tabular}




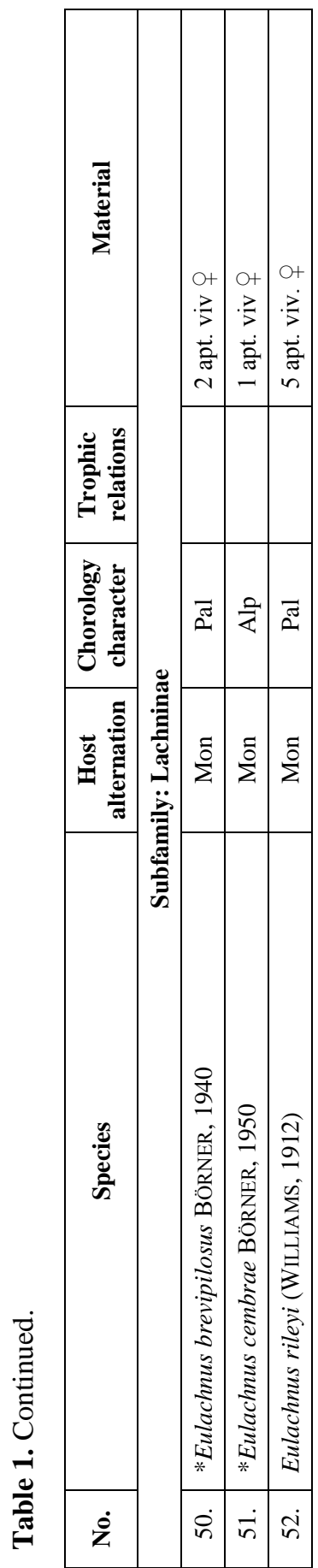


Table 2. Host plant index and associated aphid species collected in the Botanic Garden of the Jagiellonian University, Kraków.

\begin{tabular}{|c|c|c|}
\hline No. & Host plant & Aphid species \\
\hline 1. & Acer buergerianum MIQ. & Periphyllus testudinaceus \\
\hline 2. & Acer cappadocicum GLED. & Periphyllus testudinaceus \\
\hline 3. & Acer carpinifolium SIEB. et ZUCC. & Periphyllus testudinaceus \\
\hline 4. & Acer circinatum PURSH. & $\begin{array}{l}\text { Periphyllus californiensis californiensis, Periphyllus } \\
\text { testudinaceus }\end{array}$ \\
\hline 5. & Acer monspessulanum L. & Periphyllus testudinaceus \\
\hline 6. & Acer negundo L. & $\begin{array}{l}\text { Drepanosiphum platanoidis, Periphyllus aceris, } \\
\text { Periphyllus testudinaceus }\end{array}$ \\
\hline 7. & $\begin{array}{l}\text { Acer palmatum (THUNB.) } \\
\text { "Atropurpureum" }\end{array}$ & Periphyllus californiensis californiensis \\
\hline 8. & $\begin{array}{l}\text { Acer palmatum var. thunbergii } \\
\text { PAX. f. crispum }\end{array}$ & $\begin{array}{l}\text { Periphyllus californiensis californiensis, Periphyllus } \\
\text { testudinaceus }\end{array}$ \\
\hline 9. & Acer platanoides L. "Schledreri" & $\begin{array}{l}\text { Periphyllus aceris, Periphyllus californiensis } \\
\text { californiensis }\end{array}$ \\
\hline 10. & Acer platanoides L. & Periphyllus testudinaceus \\
\hline 11. & $\begin{array}{l}\text { Acer pseudoplatanus L. } \\
\text { "Purpurescens" }\end{array}$ & $\begin{array}{l}\text { Drepanosiphum platanoidis, Periphyllus californiensis } \\
\text { californiensis, Periphyllus testudinaceus }\end{array}$ \\
\hline 12. & Acer pseudoplatanus L. & Drepanosiphum platanoidis, Periphyllus testudinaceus \\
\hline 13. & Acer saccharinum L. & Periphyllus testudinaceus \\
\hline 14. & Acer saccharum MARSH. & $\begin{array}{l}\text { Periphyllus acericola, Periphyllus californiensis } \\
\text { californiensis, Periphyllus testudinaceus }\end{array}$ \\
\hline 15. & Berberis koreana PALIB. & Liosomaphis berberidis \\
\hline 16. & Betula obscura KOTSCHY. & Euceraphis punctipennis \\
\hline 17. & Betula verrucosa EHRH. "Youngii" & Euceraphis betulae \\
\hline 18. & Borago officinalis L. & Brachycaudus (Prunaphis) cardui \\
\hline 19. & Calyanthus fertilis WALT. & Rhopalosiphum padi \\
\hline 20. & $\begin{array}{l}\text { Caragana arborescens LAM. } \\
\text { "pendula" }\end{array}$ & $\begin{array}{l}\text { Therioaphis (Rhizoberlesia) riehmi, Acyrthosiphon } \\
\text { (Acyrthosiphon) caraganae caraganae }\end{array}$ \\
\hline 21. & Caragana microphylla LAM. & Therioaphis (Rhizoberlesia) riehmi \\
\hline 22. & Carpinus turczaninovii HANCE. & Myzocallis (Myzocallis) carpini \\
\hline 23. & Cornus mas L. & Anoecia (Anoecia) corni \\
\hline 24. & Cornus sanguinea L. & Anoecia (Anoecia) corni \\
\hline 25. & Cornus walteri WANGERIN & Anoecia (Anoecia) corni \\
\hline 26. & Corylus avellana L. Var. Pontica & Myzocallis (Myzocallis) coryli \\
\hline 27. & Corylus avellana L. "Warszawski" & Corylobium avellanae \\
\hline 28. & Corylus avellana L. & Corylobium avellanae \\
\hline 29. & Crataegus monogyna JACQ. & Dysaphis crataegi crataegi \\
\hline 30. & Elaeagnus umbellate THUNB. & Capitophorus elaeagni \\
\hline 31. & Euonymus hamiltoniana WALLICH. & Aphis (Aphis) fabae fabae \\
\hline 32. & $\begin{array}{l}\text { Fagus grandifolia J.F.EHRH. f. } \\
\text { pubescens FERN. et REHD. }\end{array}$ & Phyllaphis fagi \\
\hline 33. & Fagus sylvatica L. "pendula" & Phyllaphis fagi \\
\hline 34. & Fagus sylvatica $\mathrm{L}$. & Phyllaphis fagi \\
\hline 35. & Hibiscus syriacus L. & Aphis (Aphis) craccivora craccivora \\
\hline 36. & Hippophae rhamnoides L. & Capitophorus similis \\
\hline 37. & Juglans ailanthifolia CARR. & Panaphis juglandis \\
\hline 38. & $\begin{array}{l}\text { Larix gmelinii (RUPR.)RUPR. ex } \\
\text { KUZEN (= L. kurilensis MAYR.) }\end{array}$ & Adelges (Adelges) laricis \\
\hline 39. & Lilia sp. & Aphis (Aphis) fabae fabae \\
\hline
\end{tabular}


Table 2. Host plant index and associated aphid species collected in the Botanic Garden of the Jagiellonian University, Kraków.

\begin{tabular}{|c|c|c|}
\hline No. & Host plant & Aphid species \\
\hline 40. & Lonicera caerulea $\mathrm{L}$. & Rhopalomyzus (Judenkoa) lonicerae \\
\hline 41. & Lonicera korolkowii STAPF. & Myzus (Nectarosiphon) persicae \\
\hline 42. & Lonicera $x y$ losteum L. & Hyadaphis foeniculi foeniculi \\
\hline 43. & Picea abies (L.) H.KARST. & Adelges (Sacchiphantes) viridis \\
\hline 44. & Pinus cembra $\mathrm{L}$ & Eulachnus cembrae, E. rileyi \\
\hline 45. & Pinus nigra ARN. & Eulachnus rileyi \\
\hline 46. & Prunus ceracifera EHRH. & Brachycaudus (Acaudus) populi \\
\hline 47. & Prunus padus L. & Rhopalosiphum maidis \\
\hline 48. & Prunus tenella BATSCH. & Brachycaudus (Appelia) prunicola prunicola \\
\hline 49. & Quercus robur L. "Concordia" & Myzocallis (Agrioaphis) castanicola \\
\hline 50. & Quercus robur $\mathrm{L}$. & Tuberculatus (Tuberculoides) annulatus \\
\hline 51. & Ribes nigrum L. "Titania" & Hyperomyzus (Hyperomyzus) lactucae \\
\hline 52. & Ribes nigrum L. & Hyperomyzus (Hyperomyzus) pallidus \\
\hline 53. & Rosa floribunda: La palome & Macrosiphum (Macrosiphum) rosae \\
\hline 54. & Rosa lambertiane: Hybrid musk & Macrosiphum (Macrosiphum) rosae \\
\hline 55. & Rosa"La Polonia" & Macrosiphum(Macrosiphum) rosae \\
\hline 56. & Rosa borbonica & Macrosiphum (Macrosiphum) rosae \\
\hline 57. & Rosa polyatha hybrida & Macrosiphum (Macrosiphum) rosae \\
\hline 58. & Rosa rugosa THUNB. & Chaetosiphon (Pentatrichopus) tetrarhodum \\
\hline 59. & $\begin{array}{l}\text { Spiraea japonica L.F. "Anthony } \\
\text { waterer" Pl. cult. }\end{array}$ & Aphis (Aphis) spiraephaga \\
\hline 60. & Staphylea pinnata $\mathrm{L}$. & $\begin{array}{l}\text { Rhopalosiphoninus (Neorhopalosiphoninus) staphyleae } \\
\text { staphyleae }\end{array}$ \\
\hline 61. & Tilia cordata MILL. & Eucallipterus tiliae \\
\hline 62. & Tilia euchlora C. KOCH. & Eucallipterus tiliae \\
\hline 63. & Tilia platyphyllos SCOP. & Eucallipterus tiliae \\
\hline 64. & $\begin{array}{l}\text { Ulmus pumila L. var. arborea } \\
\text { LITW. }\end{array}$ & Eriosoma anncharlotteae \\
\hline 65. & Ulmus glabra HUDS. & Colopha compressa, Tinocallis (Sappocallis) saltans \\
\hline 66. & $\begin{array}{l}\text { Viburnum opulus L. "Boul de } \\
\text { Neige" }\end{array}$ & Aphis (Aphis) viburni \\
\hline
\end{tabular}

Various kinds of studies, including faunistic research, have been conducted in botanic gardens. As far as Aphidoidea diversity is concerned, only the Botanic Garden in Poznań (WILKANIEC 2004), the Dendrology Garden of the Poznan Agricultural University (SZTUKOWSKA \& WILKANIEC 2005) and the Kórnik Arboretum (RATAJCZAK \& WILKANIEC 2011) have been investigated up till now. These studies found that the species richness of aphids increased with increasing numbers of plant species in a relatively small area. The research done during four growing seasons in the Kórnik Arboretum confirmed the presence of 186 species or groups of species of aphids. However, these results are scarcely comparable with those of the present study, which was shorter (only two growing seasons). 


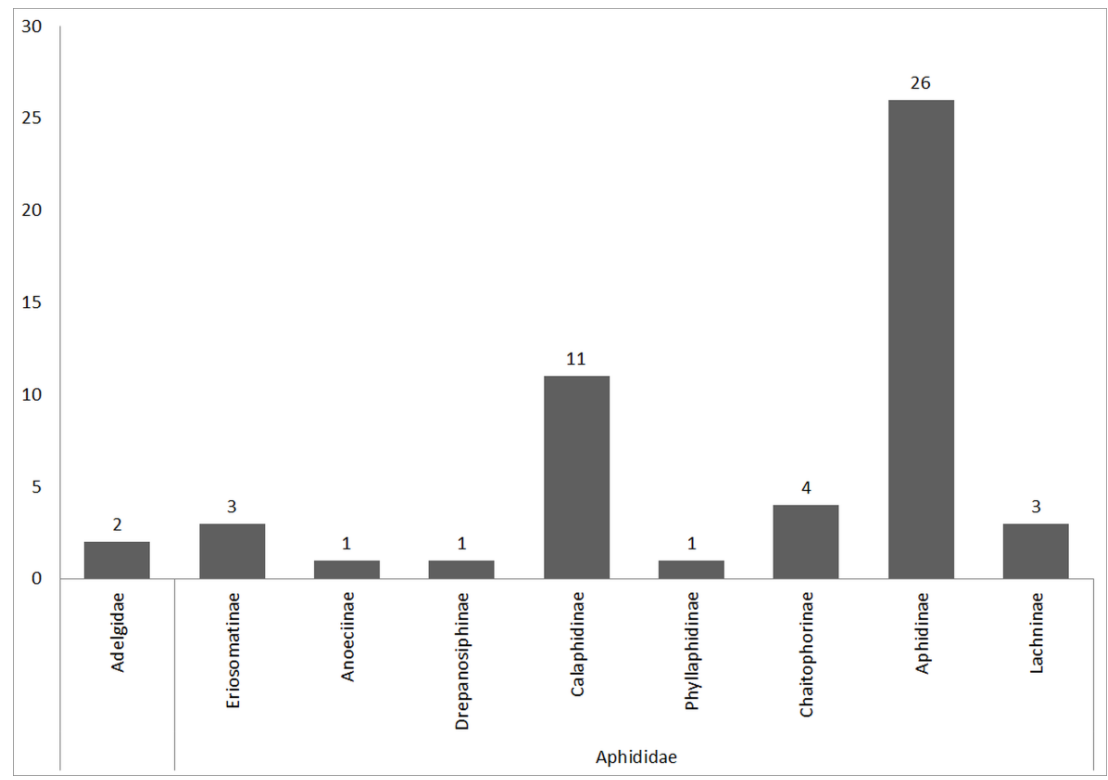

Fig. 1. Number of species from the family Adelgidae and subfamilies within Aphididae collected in the Botanic Garden of the Jagiellonian University, Kraków.

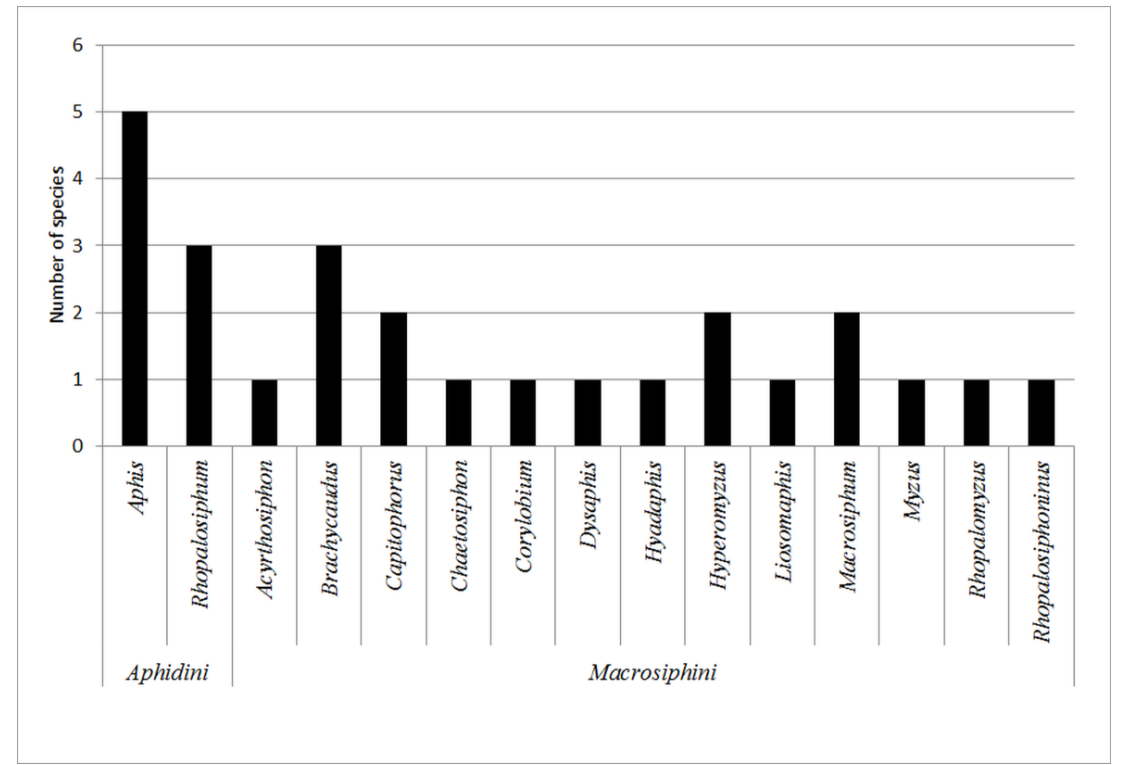

Fig. 2. Number of species in particular genera within Aphidinae collected in the Botanic Garden of the Jagiellonian University, Kraków. 


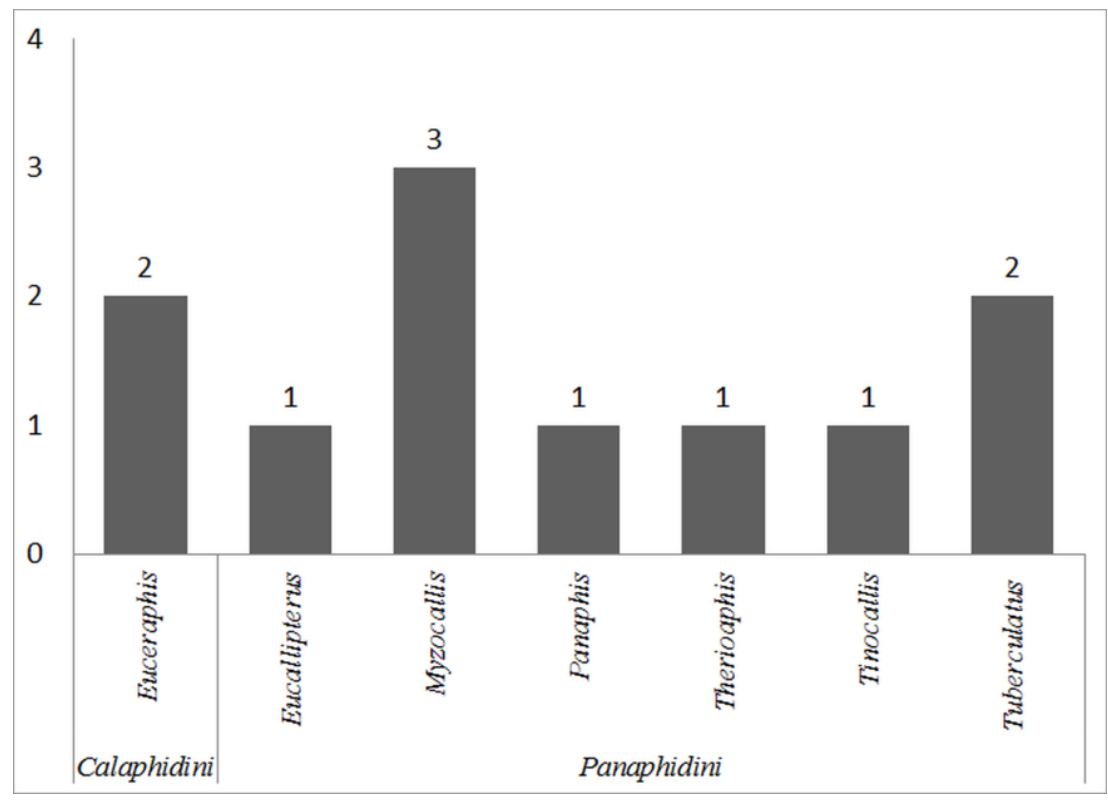

Fig. 3. Number of species in particular genera within Calaphidinae collected in the Botanic Garden of the Jagiellonian University, Kraków.

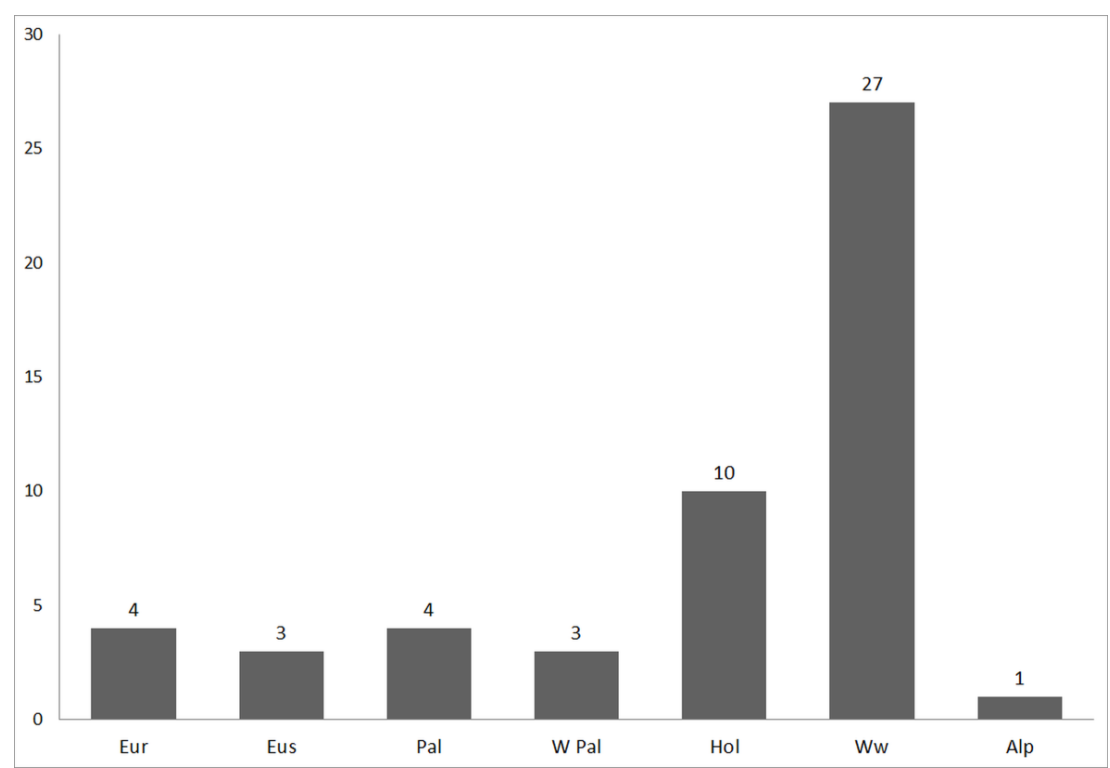

Fig. 4. Participation of chorological characters of aphids in the Botanic Garden of the Jagiellonian University, Kraków. Chorology character: Alp - Alpine, Eur - European, Eus - Euro-Siberian, Ww - worldwide, Hol - Holarctic, Pal - Palaearctic, W Pal - Western Palaearctic. 


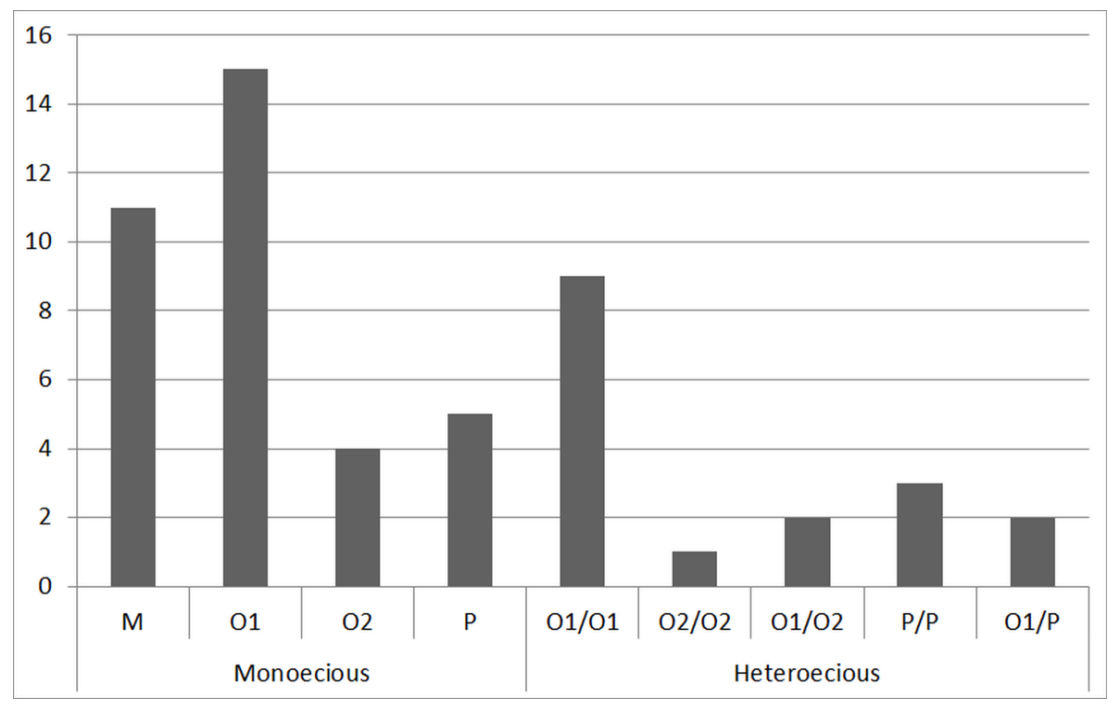

Fig. 5. Trophic structure of aphids in the Botanic Garden of the Jagiellonian University, Kraków. Trophic relations: $\mathrm{M}-$ Monophagous, $\mathrm{O} 1-1^{\text {st }}$ degree oligophagous, $\mathrm{O} 2-2^{\text {nd }}$ degree oligophagous, $\mathrm{P}$ - Polyphagous.

In addition, a variety of collecting methods were used - aphids were taken directly from their host plants as against a combination of two methods - direct collection from the host plants and Moericke traps. Using the direct method SzTUKOWSKA \& WILKANIEC (2005) collected 47 species of aphids from two families (Adelgidae and Aphididae) in the Dendrology Garden of the Poznań Agricultural University. Among Aphididae there were nine subfamilies: Anoeciinae, Aphidinae, Chaitophorinae, Drepanosiphinae, Lachninae, Myzocallidinae, Pemphiginae, Phyllaphidinae and Thelaxinae, which inhabited 26 species of trees and 38 species of shrubs. During the two seasons of research in the Poznan Botanic Garden, a total of 107 species of aphids representing the families Adelgidae, Phylloxeridae and Aphididae were collected. 36 of these species, collected directly from 82 taxa of trees and shrubs, were recorded (WILKANIEC 2004). The numbers of identified taxa in both the Poznan Botanic Garden and the Poznań Agricultural University's Dendrology Garden are similar to that in our study in the Jagiellonian University's Botanical Garden. The collection time and method were also similar, as was the number of identified host plants. However, our study did not demonstrate that there might be more species of aphids in a relatively small area with a rich floristic composition. For example, about 40 taxa of maples (Acer spp.) from various parts of the world grow in the Kraków Botanical Garden. Despite the richness of potential host plants, the dominant species associated with maples was Periphyllus testudinaceus, which was recorded in 19 samples from 13 taxa of plants 
from the genus Acer spp. Moreover, only 7 species alien to the Polish fauna were recorded: Panaphis juglandis, Tinocallis (Sappocallis) saltans, Periphyllus californiensis californiensis, Aphis (Aphis) spiraephaga, Acyrthosiphon (Acyrthosiphon) caraganae caraganae, Macrosiphum (Macrosiphum) euphorbiae and Myzus (Nectarosiphon) persicae. On the other hand, the present study yielded data on five species previously known from only one or a few localities in Poland: Eriosoma anncharlotteae, Tinocallis (Sappocallis) saltans, Brachycaudus (Acaudus) populi, Rhopalosiphoninus (Neorhopalosiphoninus) staphyleae staphyleae, including rarely collected species of the genus Eulachnus $-E$. brevipilosus and E. cembrae (KANTURSKI \& WIECZOREK 2014).

\section{REFERENCES}

ACHREMOwiCZ J. 1978. Rare and less well-known in Poland aphid species of ornamental plants. Zeszyty problemowe Postępów Nauk Rolniczych 208: 141-146. (in Polish)

ACHREMOWicz J., MAŚlAŃSKA L., OBRockA E. 1986. The research on the fauna of aphids damaging greenhouse and potted ornamental plants. Zeszyty problemowe Postępów Nauk Rolniczych 329: 54-68. (in Polish)

Coeur d’acier A., Pérez Hidalgo N., Petrović-Obradović O. 2010. Chapter 9.2. Aphids (Hemiptera, Aphididae). [in:] A. Roques, M. KenIS, D. LeES, C. LopeZ-VAamonde, W. Rabitsch, J.-Y. RAsPlus, D.B. RoY (eds.). Alien terrestrial arthropods of Europe. BioRisk 4(1): 435-474.

JUnkiert Ł., WIECZOREK K., WoJCIECHOWSKI W. 2011. Periphyllus californiensis (ShINJI, 1917) (Hemiptera: Aphidoidea) - an invasive aphid species new to Poland. Polish Journal of Entomology 80(1): 3-12.

KANTURSKI M., WIECZOREK K. 2012. Methods of sampling and preparation of aphids (Hemiptera: Aphidoidea) in faunal, taxonomic and molecular studies. Młodzi Naukowcy dla Polskiej Nauki 8(5): 137-143. (in Polish)

KANTURSKi M., WiECZOREK K. 2014. New localities of the aphids of the genus Eulachnus DEL GUERCIO, 1909 (Hemiptera: Aphididae: Lachninae) in southern Poland. Wiadomości Entomologiczne 33(1): 15-20. (in Polish)

LANKOSZ-MrÓz M. 2006. Botanic Garden of the Jagiellonian University. Part 1. Panacea 2(15): 27-29. (in Polish)

Nieto Nafría J.M, FAvret C., Akimoto S-I., Barbagallo S., Chakrabarti S., Mier Durante M.P., Miller G.L., Pérez Hidalgo N., Qiao G-X., Sano M., Stekolshchikov A., Wegierek P. 2011. Register of genus-group taxa of Aphidoidea. [in:] J.M. NIETO NAFRÍA, C. FAVRET (eds.). Registers of Family-Group and Genus-Group Taxa of Aphidoidea (Hemiptera Sternorrhyncha). Universitad de León, León, 84-404.

RAtAJCZAK J., WilkAniEC B. 2011. Aphid fauna (Hemiptera: Aphidoidea, Phylloxeroidea) in Kórnik Arboretum (Wielkopolska). Wiadomości Entomologiczne 30(1): 17-26. (in Polish) 
SZTUKOWSKA K., WiLKANIEC B. 2005. Occurrence of aphids (Hemiptera: Aphidoidea) on trees and shrubs in the Dendrological Garden of the Agricultural University in Poznan. Wiadomości Entomologiczne 24(3): 133-146. (in Polish) (in Polish)

WALKOWICZ T. 2001. Social and environmental aspects of creating and maintaining urban green areas. Fundacja Wspierania Inicjatyw Ekologicznych. Intenret: http://www.zb.eco.pl/inne/lokalne/fwie.htm (in Polish)

WieCZOREK K. 2010. A Monograph of Siphini MoRdVILKo, 1928 (Hemiptera, Aphidoidea: Chaitophorinae). Wydawnictwo Uniwersytetu Śląskiego, Katowice.

WieCZOREK K. 2011. Aphid species alien to Poland. Polish Journal of Entomology 80(2): 203-224.

WiLKANIEC B. 2004. Aphids of the Botanical Garden in Poznan. [In:] P. INDYKIEwicz, T. BARCZAK (esds.). Urban fauna of Central Europe in the 21 century. LOGO, Bydgoszcz, 167-177. (in Polish)

WOJCIECHOWICZ - ŻYTKO E., JANKOWSKA B. 2011. Aphids and their predators occurring on some shrubs in the Botanic Garden of the Jagiellonian University in Kraków. Aphids and other Hemipterous Insects 17: $145-154$.

Received: 15 August July 2015

Accepted: 10 September 2015 